\section{Kontaktallergien kratzen schon an Babys Haut}

Bislang ging man davon aus, dass Babys und Kleinkinder von Kontaktallergien weitgehend verschont bleiben. Zum einen weil das Immunsystem in diesem Alter nicht völlig ausgereift ist, zum anderen weil die Dauer der Exposition für eine Sensibilisierung zu kurz ist. Leider falsch - schon die Kleinsten trifft es!

$\mathrm{D}$ ie Autoren einer italienischen Studie unterzogen 321 Kleinkinder $(<3$ Jahre), die an einer immer wiederkehrenden Dermatitis litten, einem PatchTest und überprüften deren Reaktion auf 30 gängige Kontaktallergene, unter anderem auf Nickelsulfat.

Insgesamt 200 der getesteten Kleinkinder reagierten auf mindestens eine Substanz allergisch. Auf mehr als eine Substanz sprachen 95 Kinder an. Eine atopische Dermatitis scheint die Allergieneigung jedoch nicht zu forcieren: Die Prävalenz einer Kontaktallergie war bei Kindern mit $(61,3 \%)$ und ohne atopische Dermatitis (63\%) gleich hoch. Das Durchschnittsalter der positiv Getesteten lag bei etwa 27 Monaten. Um falsch positive Auswertungen zu vermeiden, beurteilten die Ärzte die Hautstellen zweimal, am zweiten sowie am vierten Tag. Nur wenn sich die Hautveränderungen innerhalb dieser Zeit verschlechterten, galt das Ergebnis als positiv. Kinder mit akuter Dermatitis an der Teststelle, systemischer Antihistaminika-Therapie oder lokaler Behandlung mit Kostikosteroiden waren nicht in die Studie aufgenommen worden.

Nickelsulfat war das Allergen, auf das die meisten Kinder ansprachen (26,8\%), gefolgt von Kaliumbichromat (9\%), Cocamidopropyl-Betaine $(7,2 \%)$, Kobaltchlorid (6,2\%), Neomycinsulfat (5\%), Methylchloroisothiazolinon bzw. Methylisothiazolinon
$(4,4 \%)$ und Dispersionsfarbstoffen (blau $3,1 \%$, rot $2,8 \%$, gelb $1,9 \%$ ).

Mit all diesen Kontaktallergenen scheinen die Kinder schon von Geburt an konfrontiert zu sein, so die Autoren. Nickel findet sich beispielsweise in Reißverschlüssen oder Druckknöpfen, es ist aber auch in manchen Reinigungsprodukten enthalten. Cocamidopropyl-Betaine und Methylchloroisothiazolinon bzw. Methylisothiazolinon sind Bestandteil vieler Pflegeprodukte für Babys wie Shampoos, Feuchttücher oder Cremes.

Fazit: Aufgrund der hohen Prävalenz raten die Autoren eindringlich, auch bei den Kleinsten eine Kontaktdermatitis in Erwägung zu ziehen und bei entsprechender Indikation einen Patch-Test durchzuführen, etwa wenn eine Dermatitis persistiert oder nicht auf herkömmliche Therapiemaßnahmen anspricht. Dr. Dagmar Kraus

Fortina $\mathrm{A}$ et al. Contact sensitization in very young children. JAAD 2011; 65: 772-9 